\title{
Los gonfotéridos (Mammalia, Proboscidea) de Uruguay: taxonomía, estratigrafía y cronología
}

\section{The gomphotherids (Mammalia, Proboscidea) from Uruguay: taxonomy, stratigraphy and chronology}

\author{
D. Perea ${ }^{1}$, M.T. Alberdi ${ }^{2}$ \\ 1 Instituto de Ciencias Geológicas, Facultad de Ciencias, UdelaR, Iguá 4225, 11400 Montevideo, Uruguay. Email: perea@ \\ fcien.edu.uy \\ 2 Departamento de Paleobiología. Museo Nacional de Ciencias Naturales, CSIC, José Gutiérrez Abascal, 2 28006-Madrid \\ (España). Email: alberdi.maite@gmail.com
}

\section{RESUMEN}

En Uruguay se han hallado numerosos aunque relativamente fragmentarios restos de Gomphotheriidae. Pertenecientes a esta familia existen materiales asignados a la Formación Camacho (Mioceno tardío), y a unidades del Pleistoceno y Holoceno temprano (formaciones Sopas, Libertad y Dolores). Las formaciones Camacho y Sopas contienen restos de Gomphotheridae indeterminados, mientras que la especie Stegomastodon platensis está identificada para las formaciones Libertad y Dolores. Entendemos taxonómicamente adecuado el criterio de mantener a los gonfotéridos habitantes de las planicies sudamericanas bajo el género Stegomastodon y considerar dicha especie como única representante del género en Sudamérica.

Palabras clave: Gomphotheriidae; Stegomastodon platensis; Uruguay; Mioceno tardío; Pleistoceno-Holoceno temprano.

\section{ABSTRACT}

A large sample composed of relatively fragmentary remains of Gomphotheriidae was found in Uruguay. Belonging to this family there are materials assigned to Camacho Formation (Late Miocene), and to Pleistocene to Early Holocene units (Sopas, Libertad and Dolores formations). The Camacho and Sopas formations contain remains of indeterminate Gomphotheriidae, and the species Stegomastodon platensis is identified for the Libertad and Dolores formations. We think taxonomically suitable to support the opinion that all gomphotherids inhabitants of South American plains pertain to the genus Stegomastodon and to consider that species as the only representative of the genus in South America.

Keywords: Gomphotheriidae; Stegomastodon platensis; Uruguay; Late Miocene; Pleistocene-Early Holocene. estratigrafía y cronología. Estudios Geológicos 71(2): e036. http://dx.doi.org/10.3989/egeol.41864.346.

Copyright: (c) 2015 CSIC. This is an open-access article distributed under the terms of the Creative Commons Attribution-Non Commercial (by-nc) Spain 3.0 License. 


\section{Introducción}

Los “mastodontes" de Uruguay están representados por numerosos restos provenientes de variadas localidades geográficas y con un amplio registro estratigráfico. Lamentablemente, muchos de los ejemplares estudiados son muy fragmentarios lo que dificulta su análisis taxonómico. A esto a veces se agrega también una ubicación estratigráfica imprecisa. Un excepción a esto lo constituyen restos craneanos y mandibulares recientemente hallados, asignados a Stegomastodon waringi y detalladamente descritos por Gutiérrez et al. (2005) y Alberdi et al. (2007), provenientes de las formaciones Libertad y Dolores respectivamente, los cuales no se incluyen en la presente contribución.

La mayor parte de los trabajos anteriores a los arriba citados son referencias someras a diversos materiales de mastodontes de Uruguay y no consisten en revisiones sistemáticas ni descripciones detalladas. Un importante conjunto de estos trabajos están citados en Mones \& Francis (1973).

En el presente trabajo se describe dicho material, referido a diferentes taxa dentro de Gomphotheriidae en obras anteriores a las descripciones de Gutiérrez et al. (2005) y Alberdi et al. (2007), así como otros ejemplares procedentes de Uruguay no considerados en ningún trabajo previo. Asimismo se discute el peculiar registro de gonfotéridos en la Formación Camacho y algunos aspectos de la taxonomía utilizada para los mastodontes sudamericanos.

\section{Material y métodos}

Los gonfotéridos estudiados se encuentran depositados en las siguientes colecciones de Uruguay: Facultad de Ciencias-Universidad de la República, Montevideo (FC-DPV); Museo Nacional de Historia Natural de Montevideo (MNHN); Museo "Bautista Rebuffo" (MBR) y Museo Paleontológico "Armando Calcaterra" (MPAC), Colonia; Museo Paleontológico "Francisco L. Roselli", Nueva Palmira (MPFLR); Museo Paleontológico "Alejandro Berro", Mercedes (MPAB).

Para la taxonomía se siguieron los criterios de Alberdi et al. (2002) y Prado et al. (2005) a diferencia de Mothé et al. (2012) y Lucas (2013), en el entendido que al momento no existen suficientes elementos de juicio concretos para diferenciar a nivel genérico los restos asignables a Stegomastodon procedentes de Sudamérica de los de Norteamérica, por lo que el género Notiomastodon no exhibe caracteres morfológicos diagnósticos claros ni suficientes que justifiquen su distinción de aquél (Mothé et al., 2012; ver discusión y conclusiones).

El carácter comúnmente utilizado para la separación de Stegomastodon platensis (Ameghino, 1888) respecto de $S$. waringi (Holland, 1920) fue el mayor grado de complejidad de los lofos de los molares que distinguen al primero (Gutiérrez et al., 2005; Alberdi et al., 2007). Este criterio resulta muy endeble, por lo que entendemos más adecuado considerar dentro del género una única especie, $S$. platensis al igual que Mothé et al. (2012; ver discusión y conclusiones).

Se siguieron las propuestas de la bioestratigrafía regional sugeridas por Cione \& Tonni (2005) y la estratigrafía del Cuaternario de Uruguay actualizada por Ubilla \& Perea (1999) y Ubilla et al. (2009). Las unidades litoestratigráficas que se han confirmado como claramente contentivas de gonfotéridos en Uruguay son las formaciones Camacho y Libertad, ambas definidas por Goso \& Bossi (1966); y las formaciones Sopas, definida por Antón, y Dolores, definida por Goso (Martínez \& Ubilla, 2004) (Fig. 1).

Las medidas y sus abreviaturas siguen los criterios de Gutiérrez et al. (2005) y Alberdi et al. (2007) (Fig. 2).

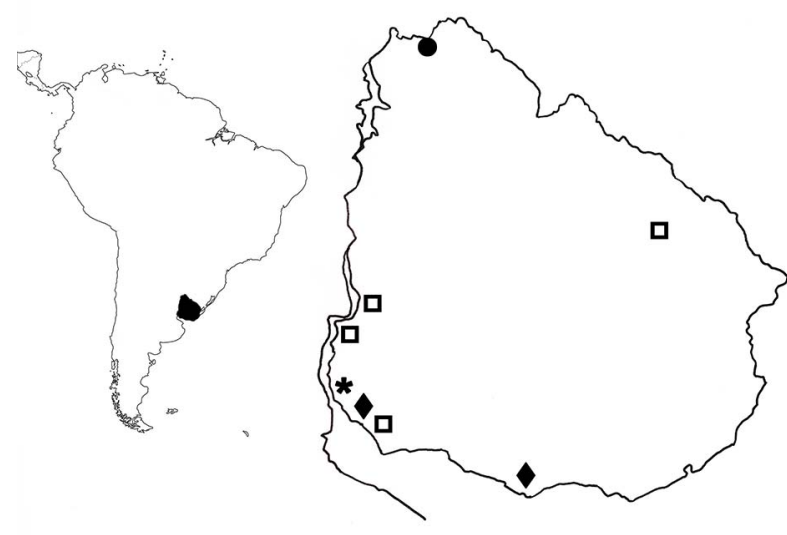

Gomphotheriidae g. et sp. indet. * Formación Camacho, Mioceno tardío; - Formación Sopas, Pleistoceno tardío.

Stegomastodon platensis $\$$ Formación Libertad, Pleistoceno; 口 Formación Dolores, Pleistoceno tardío-Holoceno temprano.

Fig. 1.-Principales localidades con gonfotéridos de Uruguay. 


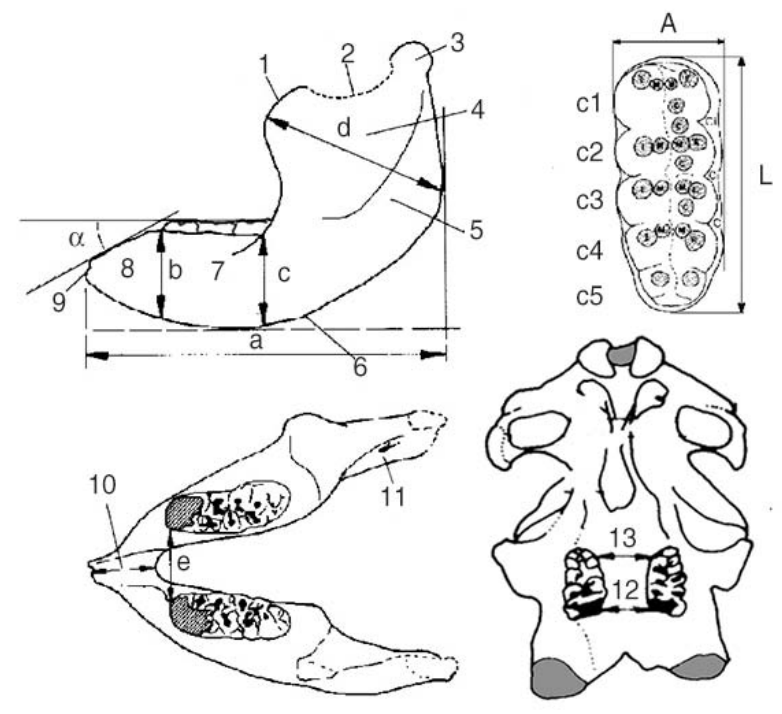

Fig. 2.-Esquema con la especificación de las medidas tomadas.

\section{Taxonomía y descripción}

Mammalia Linnaeus, 1758

Proboscidea Illiger, 1811

Gomphotheriidae Cabrera, 1929

Stegomastodon Pohlig, 1912

\section{Stegomastodon platensis (Ameghino, 1888)}

Material: MNHN-792, fragmento de cráneo con un D3 y la huella del alvéolo del D2, juvenil (Fig. 3, tabla 1); departamento de Río Negro, Arroyo Gutiérrez Grande; Formación Dolores.

Descripción: el fragmento da la idea de un cráneo bastante elevado, quizás por su condición de juvenil. El D3 de tres lofos muestra figuras de tréboles complicados con mediano grado de desgaste.

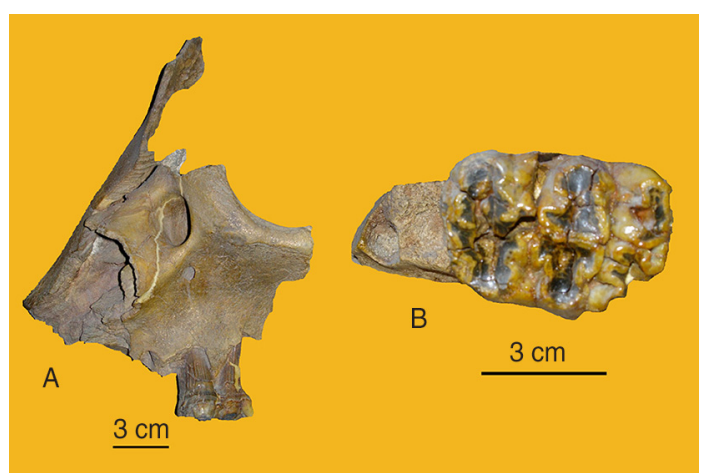

Fig. 3.-MNHN-792, A: fragmento de cráneo con un molar deciduo (D3); B: detalle de la superficie oclusal del D3 y de la huella del alvéolo del D2.
Tabla 1.-Medidas de cráneo y dentición superior, $\mathbf{m m}$. Por abreviaturas ver materiales y métodos y fig. 2

\begin{tabular}{lcccc}
\hline & MNHN-792 & MPAC-1313 & MPAB-217 & MPFLR-s/n \\
\hline A D3 & 44,6 & & & \\
L D3 & 59,1 & & & \\
12 & & 110 & & 97 \\
13 & 124,5 & & \\
A M2 & & & 93,5 & \\
L M2 & & & 123 & \\
A M3 & & 86,5 & 95 & \\
L M3 & & & 190 & \\
Ac2 M2 & & & 90 & \\
Ac3 M2 & & & 90 & \\
A 1 M3 & & & 94,5 & \\
Ac2 M3 & & & 89,5 & \\
Ac3 M3 & & & 81 & \\
Ac4 M3 & & & 77,5 &
\end{tabular}

Comentarios: este material fue dado a conocer en forma preliminar por Perea \& Martínez (1984). Existen dataciones por ${ }^{14} \mathrm{C}$ en madera que ubican los sedimentos portadores de este ejemplar en el entorno de 11000-12000 años de antigüedad (Ubilla \& Perea, 1999).

Material: MPAC-1313, porción del paladar con los dos terceros molares, muy desgastados y deteriorados (Fig. 4, tabla 1); departamento de Colonia, Barranca del Arroyo El Caño; "Pleistoceno Superior (pardo rojizo)", Formación Dolores.

Descripción: a pesar del desgaste en los molares se pueden observar láminas de esmalte sinuosas y coneletes externos. En el molar izquierdo se puede apreciar el esmalte algo rizado e incluso el final de la línea media.

Material: MPAB-217, fragmento de paladar con molares, mandíbula incompleta (Figs. 5-6, tablas 1 y 2 ) y defensa de un mismo

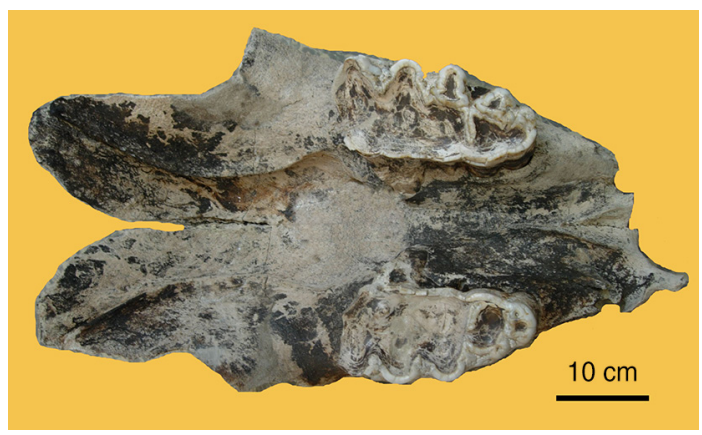

Fig. 4.-MPAC-1313, porción del paladar con los dos terceros molares. 


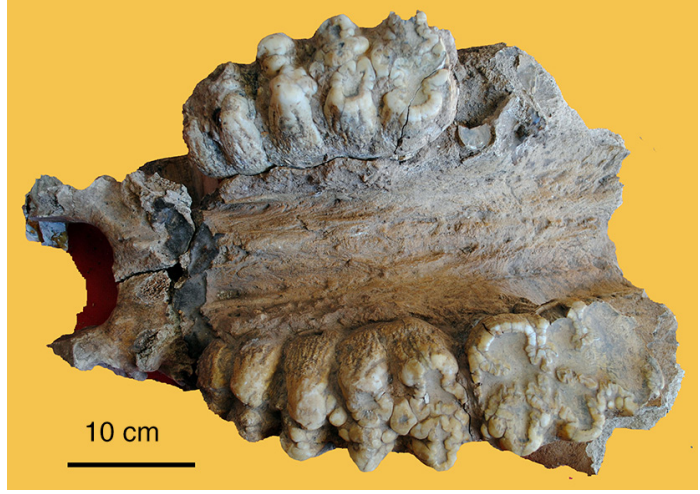

Fig. 5.-MPAB-217, paladar con molares.

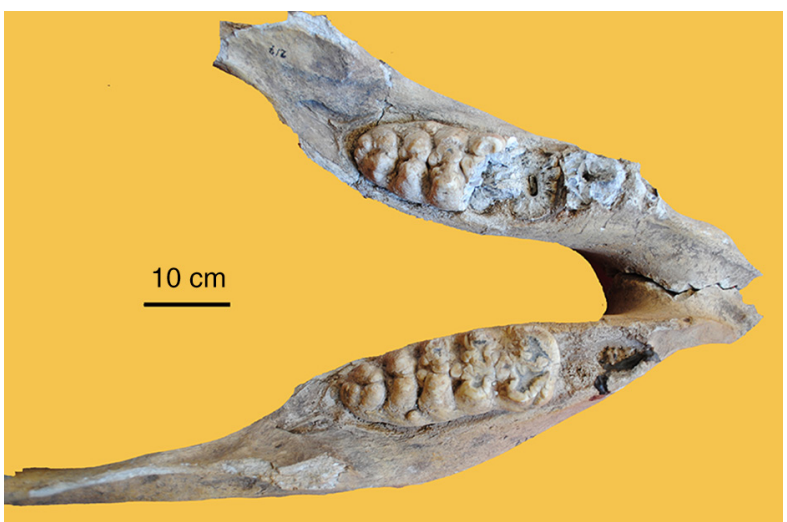

Fig. 6.-MPAB-217, mandíbula incompleta.

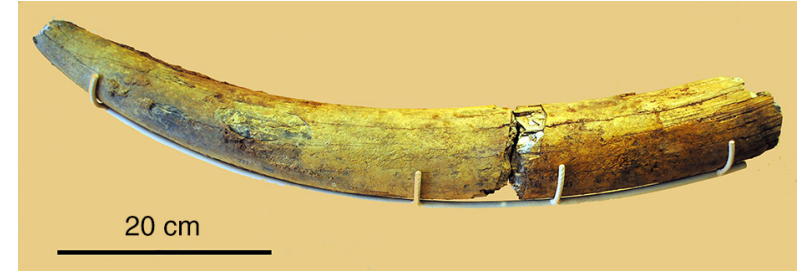

Fig. 7.-MPAB-217, defensa incompleta.

individuo (Fig. 7); departamento de Soriano, Arroyo Perico Flaco, campo de la viuda de Cannard; "Pampeano Superior Lacustre", Formación Dolores.

Descripción: El paladar presenta M2 izquierdo bastante desgastado mesialmente y los M3 con un comienzo de desgaste en el primer lofo y algo menos en el segundo, ambos molares presentan tréboles complejos y coneletes accesorios en los valles, y se observa la línea media. La mandíbula conserva los restos de de la raíz y el alveolo de los $\mathrm{m} 2$ y los $\mathrm{m} 3$ con 5 colinas y talón reducido, al izquierdo le faltan las dos primeras colinas y el derecho está entero con las dos primeras colinas algo desgastadas donde se observa figuras treboladas complicadas, en el resto se observa la línea media. La mandíbula está bastante entera pero le falta la mayor parte de las ramas ascendentes. La defensa mide casi un metro. Es robusta y curva y no presenta torsión ni banda de esmalte.

Material: MPFLR-390, defensa incompleta, con una longitud aproximada de $1000 \mathrm{~mm}$ y un diámetro máximo aproximado de $120 \mathrm{~mm}$ en la base (Fig. 8); departamento de Soriano, Arroyo Arenal Grande; Formación Libertad.

Descripción: robusta y curva, sin banda de esmalte ni torsión.

Material: MPFLR-s/n, porción de paladar con dos molares incompletos (Fig. 9, tabla 1); departamento de Colonia, en el

Tabla 2.-Medidas de mandíbulas y dentición inferior, mm. Abreviaturas como en materiales y métodos y fig. 2

\begin{tabular}{|c|c|c|c|c|c|c|}
\hline & MPAB-4021 & MPAB-217 & MBR-S/N & FC-DPV-1886 & MNHN-909 & MPFLR-408a \\
\hline a & 701 & & & & & \\
\hline b & 132,5 & 170 & 135 & & & 150 \\
\hline c & 140 & 129,5 & 125 & & & 120 \\
\hline e & & 106,5 & 80 & & & \\
\hline$\alpha$ & $25^{\circ}$ & & $30^{\circ}$ & $30^{\circ}$ & & \\
\hline 10 & 116 & & & & & \\
\hline A m2 & & 93,5 & & & & \\
\hline L m2 & & 123 & & & & \\
\hline A m3 & & 83 & 70 & & 84,5 & 87,5 \\
\hline L m3 & & 215 & 185 & & 239 & \\
\hline Ac1 $\mathrm{m} 3$ & & 82,5 & 80 & & 81,3 & 86,5 \\
\hline Ac2 $\mathrm{m} 3$ & & 80,5 & 78 & & 82,8 & 85,5 \\
\hline Ac3 m3 & & 80 & 80 & & 81,5 & 83,7 \\
\hline Ac4 m3 & & 77 & 72 & & 78,7 & \\
\hline Ac5 m3 & & & 65 & & 61 & \\
\hline
\end{tabular}




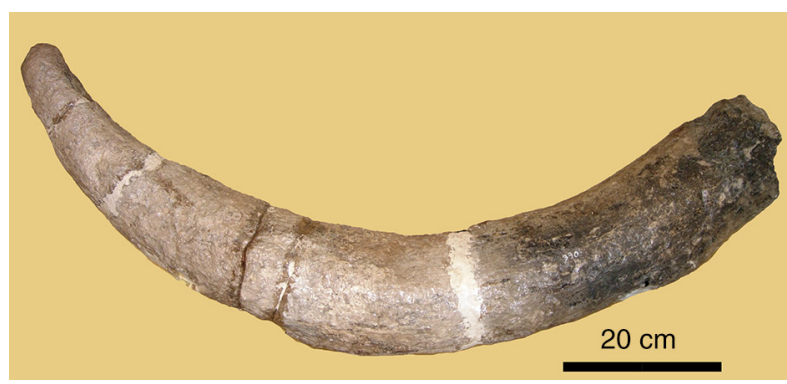

Fig. 8.-MPFLR-390, defensa incompleta.

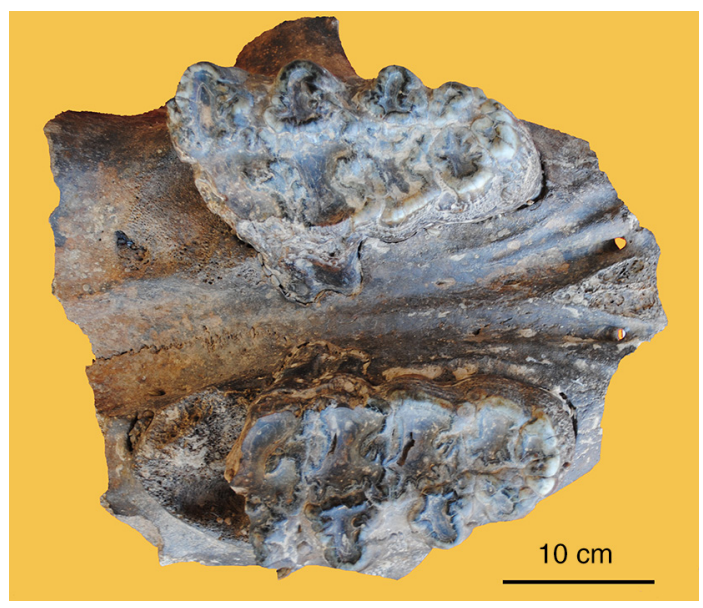

Fig. 9.-MPFLR-s/n, porción de paladar con dos molares incompletos.

lecho del Río de la Plata, sobre Punta Martín Chico; procedencia estratigráfica incierta.

Descripción: el material presenta los M3, con cinco lofos y talón reducido le falta el primer lofo y se observa muy bien en el M3 derecho, el esmalte muy plegado configurando tréboles complejos, la superficie oclusal está bastante desgastada.

Material: MBR-s/n en vitrina, mandíbula con las regiones articular y coronoides muy deterioradas (están rotas) presenta ambos $\mathrm{m} 2$ muy desgastados y ambos $\mathrm{m} 3$ (Fig. 10, tabla 2); departamento de Colonia, Arroyo San Pedro; procedencia estratigráfica incierta.

Descripción: mandíbula robusta; borde inferior curvo; sínfisis medianamente aguzada. Los $\mathrm{m} 3$ son largos con 5 lófidos y talón, el m3 izquierdo está menos desgastado que el derecho, los tres primeros lófidos presentan tréboles complejos y se observa bien la línea media.

Material: FC-DPV-1886, mandíbula incompleta, bastante deteriorada, conserva un fragmento de la rama mandibular izquierda con el tercer molar muy desgastado y deteriorado, la sínfisis y el inicio de la rama mandibular derecha donde se observa el alveolo del diente correspondiente (Fig. 11, tabla 2); departamento de Soriano, Estancia "La Perdiz"; procedencia estratigráfica incierta.

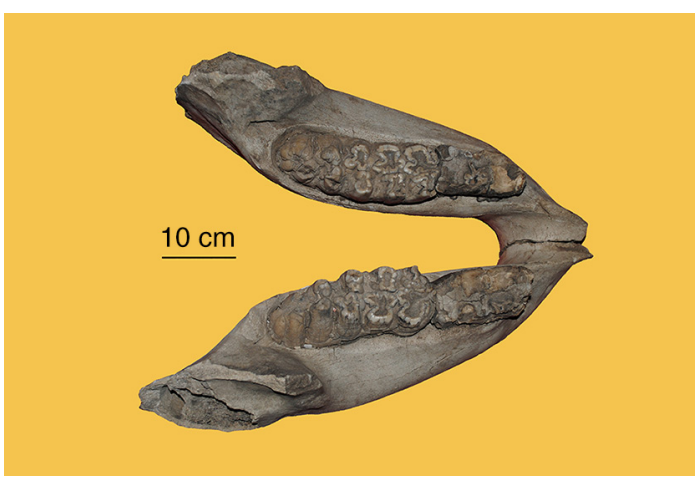

Fig. 10.-MBR-s/n, mandíbula.

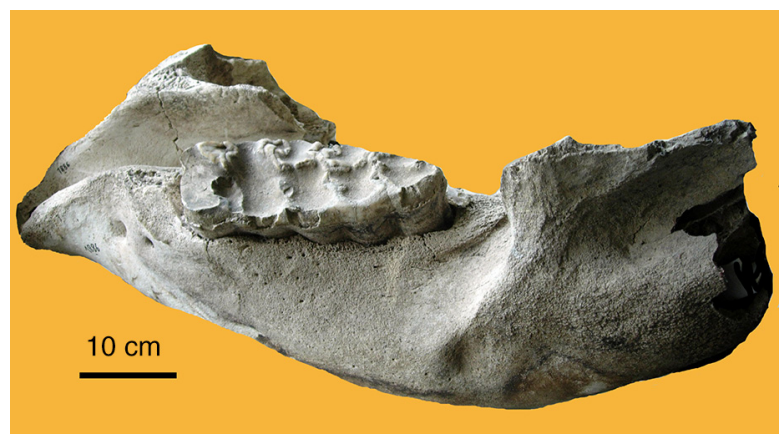

Fig. 11.-FC-DPV-1886, mandíbula incompleta.

Descripción: mandíbula robusta, con borde inferior recto hasta el m3, curvándose luego hacia arriba. A pesar del grado de desgaste y el deterioro que presentan, se observa cierto grado de complejidad en los tréboles.

Material: MPFLR-408a, fragmento de hemimandíbula izquierda muy reconstruida, con $\mathrm{m} 3$ (Fig. 12, tabla 2); departamento de Colonia, Martín Chico, El Manzano; Formación Libertad.

Descripción: aunque muy deteriorado se observa un diente robusto y con figuras complicadas en los lófidos. Se pueden observar 5 lófidos con tréboles bien marcados y complejos, así como coneletes accesorios.

Material: MNHN-909, m3 inferior (Fig. 13, tabla 2); departamento de Lavalleja, $7^{\mathrm{a}}$ Sección, Arroyo Sauce, Ruta 8, km 163, 500, Estancia Perdomo; procedencia estratigráfica incierta.

Descripción: molar muy elongado, con cinco lófidos bien acusados y talón reducido; presenta un comienzo de desgaste en el primer lófido que apunta figuras de esmalte sinuosas y abundantes coneletes internos. Se observa una capa de cemento fuerte bordeando prácticamente todos los cónulos y coneletes y cíngulo mesial.

\section{Gomphotheriidae g. et sp. indet.}

Material: MPAB-4021, mandíbula con los dos m3 (Fig. 14, tabla 2); departamento de Soriano, Arroyo Curupí, campo "Don Lisandro"; "Pampeano Superior Lujanense". 


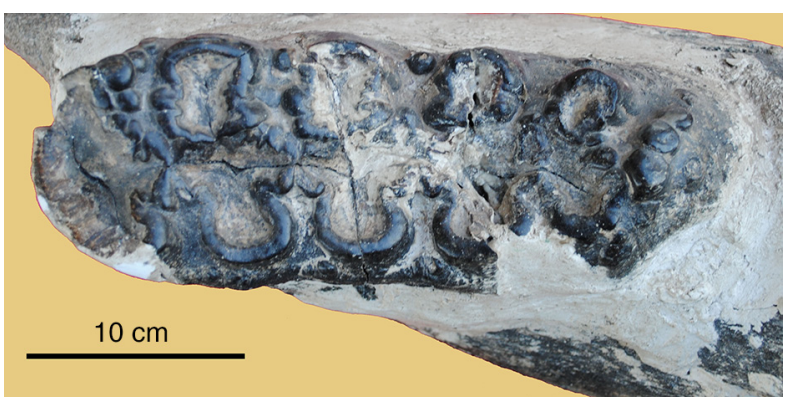

Fig. 12.-MPFLR-408a, fragmento de hemimandíbula izquierda con molar.

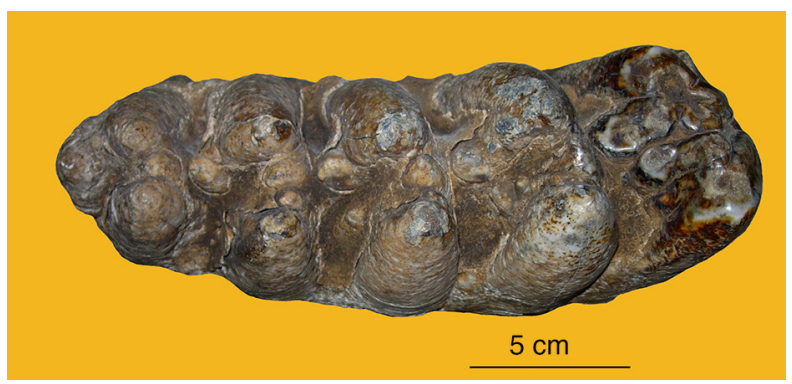

Fig. 13.-MNHN-909, m3 inferior.

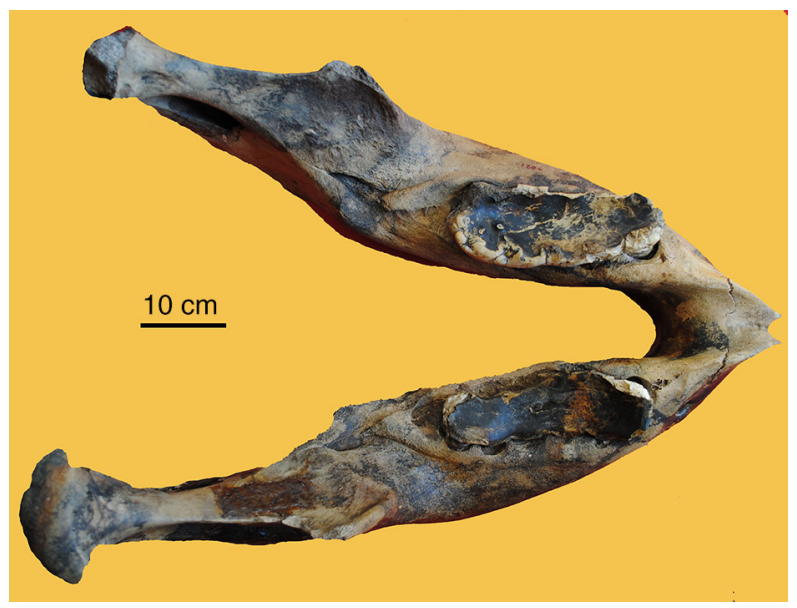

Fig. 14.-MPAB-4021, mandíbula, vista oclusal.

Descripción: mandíbula grácil; borde inferior con curvatura continua desde el extremo anterior hasta los cóndilos conservando completo el cóndilo derecho; el proceso coronoide está roto en ambas ramas mandibulares; sínfisis aguda.

Comentarios: se trata de un individuo muy viejo con un avanzado desgaste de los molares, lo cual no permite observar las principales características morfológicas dentarias. Los $\mathrm{m} 3$ carecen, por rotura, de esmalte en varias partes lo cual no permite efectuar medidas excepto la longitud total. De acuerdo a los depósitos aflorantes en el Arroyo Curupí, el "Pampeano

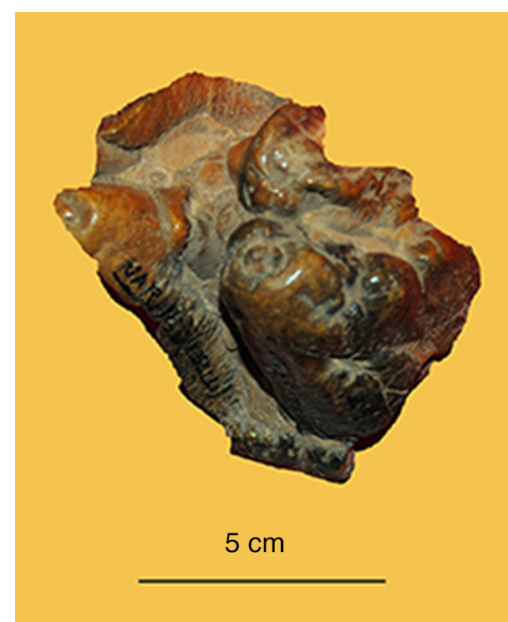

Fig. 15.-MPFLR-290, fragmento de molar.

Superior Lujanense" que figura en el catálogo del museo, puede corresponderse con la Formación Dolores.

Material: MPFLR-290, fragmento de molar (Fig. 15); departamento de Colonia, Cantera Narbona (Camacho); Formación Camacho (Fig. 16).

Descripción: el fragmento de molar muestra al menos tres conos y tres coneletes bien marcados y con escaso grado de desgaste que corresponde a un diente claramente bunodonto (gonfoterino).

Comentarios: Roselli (1976), define con este ejemplar una nueva especie Cuvieronius narancioi, pero el material, un fragmento de molar (Fig. 15), no resulta lo suficientemente diagnóstico como para trascender una determinación a nivel genérica. En la misma obra este autor describe y figura dos fragmentos más, con la misma procedencia, que asigna a la misma especie, pero no consigna su numeración de catálogo. La única pieza que se pudo observar de estas tres fue el ejemplar MPFLR-290.

Material: MNHN-57: fémur izquierdo (Fig. 17, tabla 3); departamento de Canelones, Arroyo Canelón Chico; procedencia estratigráfica incierta.

Descripción: fémur de mediana robustez, bien conservado, con fosa trocantérica medianamente acusada y epicóndilos sobresalientes; cabeza del fémur entera.

Material: MPAB- 914: fémur izquierdo (Fig. 18, tabla 3); departamento de Paysandú, Arroyo Mburucayupí; "Pampeano superior", por su procedencia geográfica es probable su asignación tentativa a la Formación Dolores.

Descripción: fémur largo y robusto, con fosa trocantérica muy marcada y cabeza bien conservada. Podría corresponder a un animal relativamente joven.

Material: MPAB-4001, fémur derecho (Fig. 19, tabla 3); departamento de Soriano, Arroyo Coquimbo; procedencia estratigráfica incierta, aunque por su procedencia geográfica es probable su asignación tentativa a la Formación Dolores.

Descripción: fémur muy grácil, con trocánter mayor poco marcado y fosa trocantérica levemente acusada. Corresponde a un animal joven y algo rodado, la cabeza se conserva bastante bien. 


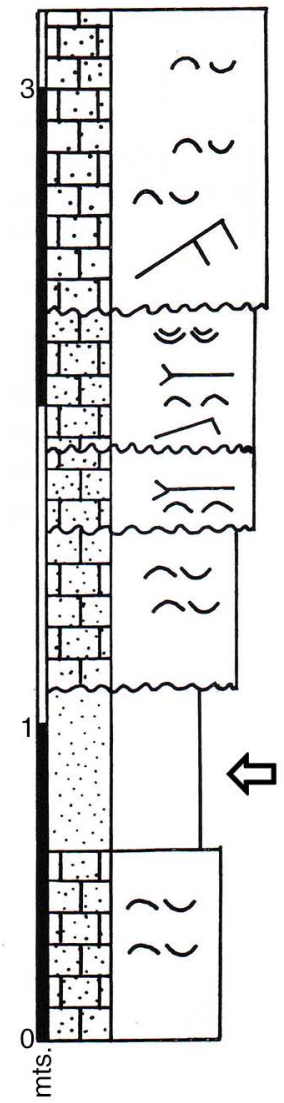

Arenisca gruesa, consolidada, cemento calcáreo, con moldes en disposición caótica. Estratificación cruzada en la base, $95 \mathrm{~cm}$

Arenisca media a gruesa, consolidada, cemento calcáreo con estratificación cruzada y moldes de bivalvos, $45 \mathrm{~cm}$

Arenisca media a gruesa, consolidada, cemento calcáreo con acuñamientos y moldes de bivalvos, base erosiva, $25 \mathrm{~cm}$

Arenisca media consolidada, cemento calcáreo, con moldes de bivalvos en posición caótica, base erosiva, $50 \mathrm{~cm}$

Arenisca muy fina, friable, blanca, $50 \mathrm{~cm}$

Arenisca fina a media, consolidada, cemento calcáreo con moldes de bivalvos en posición caótica, $60 \mathrm{~cm}$

Fig. 16.-Perfil estratigráfico esquemático de las areniscas de la Formación Camacho (Mioceno tardío) en el lugar de hallazgo del material MPFLR-290. La flecha indica el punto de colecta según Roselli (1976). Modificado de Martínez (1994).

\section{Discusión y conclusiones}

A pesar de las observaciones de Roselli (1976), consideramos a Cuvieronius narancioi un taxón no válido, ya que las piezas descritas para definir la especie, fragmentos de molariformes, no tienen suficiente valor diagnóstico para poder determinar un género o especie. Aunque, según expresa el autor, no parece haber dudas en cuanto a la procedencia estratigráfica de los materiales en cuestión. Las piezas fueron extraídas de niveles arenosos de la Cantera Narbona o Camacho (Fig. 16), Colonia, donde afloran únicamente secciones estratigráficas típicas del Miembro Cerro Bautista de la Formación Camacho (ver Martínez, 1994; Perea \& Martínez, 2004). La probable presencia de un indudable gonfotérido en esta unidad permite efectuar consideraciones interesantes que apuntan al ingreso muy temprano de esta familia a Sudamérica o a proponer una edad más tardía para la Formación Camacho, o por lo menos para su Miembro Cerro Bautista. Una hipótesis alternativa podría ser considerar estos restos como colados en la Formación Camacho y provenientes de depósitos más modernos que se erosionaron en esa localidad. Aunque muy cuestionado en lo que respecta a su taxonomía y estratigrafía (ver Lucas, 2013), existe también otro registro de Gomphotheriidae asignado por Campbell et al. (2000) al Mioceno tardío de Sudamérica. Este último ha sido discutido entre otros por Alberdi et al. (2004) y Ferretti (2008).

Aparte de este dato puntual y extremadamente curioso, el resto de los gonfotéridos de Uruguay con 


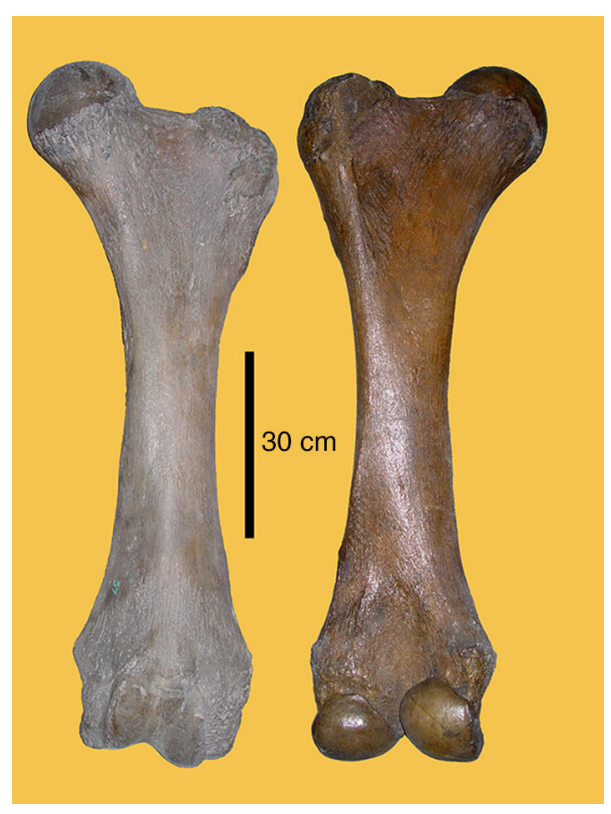

Fig. 17.-MNHN-57, fémur izquierdo, vistas anterior y posterior.

datos estratigráficos precisos proceden indudablemente de unidades del Cuaternario. Los restos más completos provienen de la Formación Dolores (Alberdi et al., 2007) y la Formación Libertad (Gutiérrez et al., 2005; Corona et al., 2012). Para la Formación Sopas se citan restos incompletos asignados a gonfotéridos indeterminados (Ubilla et al., 2009).

En el presente trabajo se mantiene el criterio taxonómico conservador de mantener a los gonfotéridos de las tierras bajas sudamericanas dentro del género Stegomastodon. Esto se basa en el entendido que los caracteres morfológicos que sustentan la distinción entre dicho género y Notiomastodon Cabrera, 1929, no resultan al momento contundentes. Los caracteres diagnósticos considerados para distinguir a ambos (Mothé et al., 2012; Lucas, 2013), como algunos caracteres del esqueleto postcraneano, el grado de graviportalidad y el número de lófidos en algunos molares, resultan taxonómicamente más consistentes con una separación a nivel específico o hasta posiblemente subespecífico. En este marco y

Tabla 3.—Medidas de fémures, $\mathrm{mm}$

\begin{tabular}{lccc}
\hline & MNHN-57 & MPAB-914 & MPAB-4001 \\
Longitud & 1003 & 1020 & 880 \\
\hline Ancho máximo proximal & 390 & 370 & 330 \\
Ancho máximo distal & 270 & 280 & 220 \\
\hline
\end{tabular}

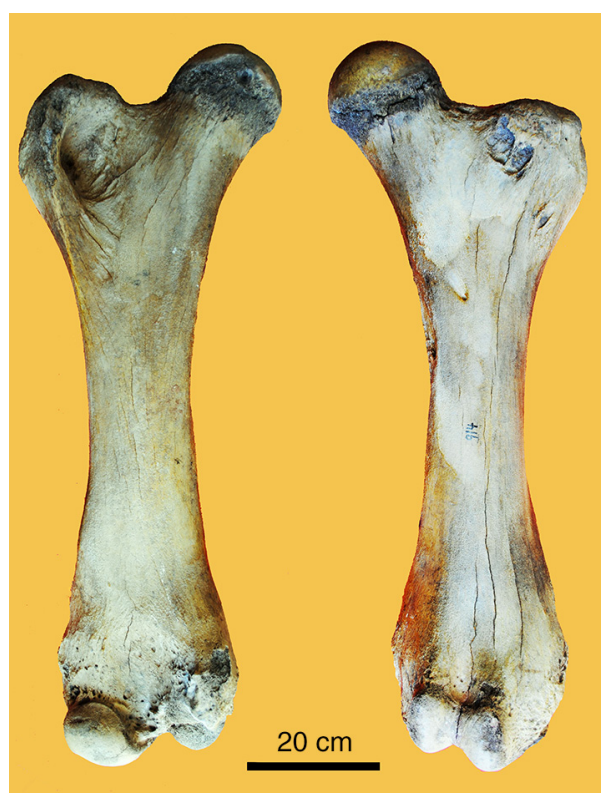

Fig. 18.-MPAB-914, fémur izquierdo, vistas posterior y anterior.

comparativamente, es mucho más evidente la identificación de Cuvieronius como entidad genérica que la diferenciación morfológica de Stegomastodon respecto de Notiomastodon. Los criterios biogeográficos y filogenéticos (Mothé et al., 2013; Lucas, 2013) aportan significativamente a la discusión de la posible validez de Notiomastodon, pero entendemos que la morfología debe ser un factor preponderante

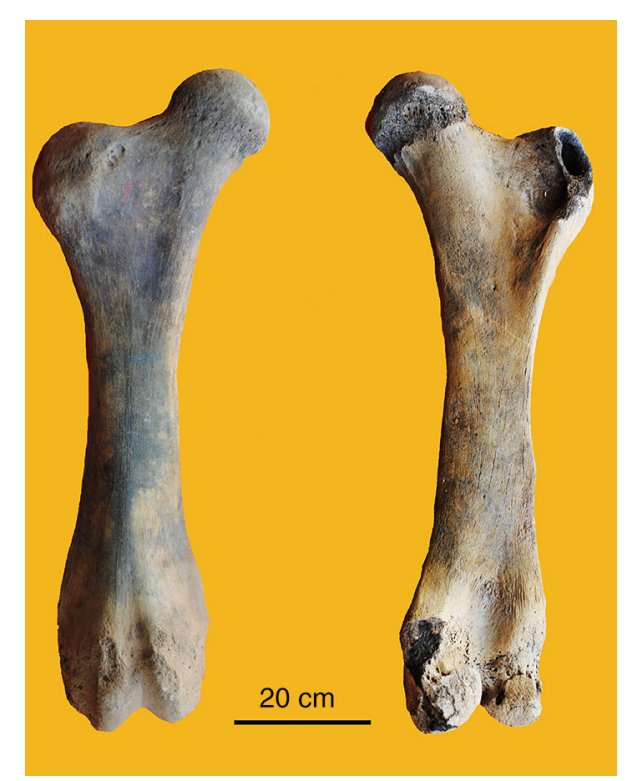

Fig. 19.-MPAB-4001, fémur derecho, vistas anterior y posterior. 
para definir la verdadera identidad de ese género, ya que la sistemática del grupo es compleja y su registro fósil en América Central y Sudamérica es aún bastante insuficiente comparado con Norteamérica (Lucas \& Alvarado, 2010).

El mayor grado de complejidad de los lofos/lófidos de los molares ha sido un elemento para separar dos especies dentro de Stegomastodon (Gutiérrez et al., 2005; Alberdi et al., 2007). Ya que hay similares niveles de complejidad de los lofos distinguibles en muestras atribuibles a diferentes poblaciones asignadas a una $\mathrm{u}$ otra de las especies sudamericanas, $S$. platensis y $S$. waringi, consideramos que lo más adecuado es reconocer una única especie válida dentro del género, $S$. platensis siguiendo a Mothé et al. (2012).

De los datos precedentes y la presente revisión se desprende una importante representación en la Formación Dolores de integrantes de Stegomastodon platensis. Dicha unidad se distribuye principalmente al sur y litoral oeste y noreste de Uruguay y los datos cronológicos obtenidos en depósitos al sur, en la Cuenca de Santa Lucía la ubican en una antigüedad de entre 30.000 y 8.000 años (Ubilla et al., 2011).

Con respecto a la Formación Libertad, existe una mandíbula asignada a $S$. waringi (Gutiérrez et al., 2005; Corona et al., 2012), para la cual se cita una datación radiométrica en base a ${ }^{14} \mathrm{C}$ AMS de 17.620+100 AP. No obstante, tenemos nuevos datos más recientes realizados sobre esmalte de diente de Toxodon ver Corona et al. (2012). Por el momento, no existen datos cronoestratigráficos claros para esta unidad dentro del Cuaternario, aunque en principio se supone más antigua que la Formación Dolores (Martínez \& Ubilla, 2004).

La Formación Sopas representa el Pleistoceno superior teniendo en cuenta su fauna, pero podría abarcar niveles más antiguos, hasta del Pleistoceno medio, atendiendo a algunas dataciones basadas en termoluminiscencia (Ubilla et al., 2009).

En suma, se convalida el registro de gonfotéridos en la Formación Camacho (Mioceno tardío), aunque restan elementos más contundentes para dar precisión a este dato, y se confirma la presencia de la familia en la Formación Sopas (Pleistoceno tardío) y de Stegomastodon platensis en las formaciones Libertad (Pleistoceno) y Dolores (Pleistoceno tardíoHoloceno temprano).

\section{AGRADECIMIENTOS}

Este trabajo ha sido posible con una beca otorgada a uno de los autores (DP) por la Fundación Carolina, España y UdelaR, Uruguay y es una contribución a los proyectos CGL2010-19116/ BOS de la DGICYT, España y ANII-FCE-2011-6752, Uruguay.

\section{Referencias}

Alberdi, M.T.; Prado, J.L. \& Cartelle, C. (2002). El registro de Stegomastodon (Mammalia, Gomphotheriidae) en el Pleistoceno superior de Brasil. Revista Española de Paleontología, 17 (2): 217-235.

Alberdi, M.T.; Prado, J.L.; Perea, D. \& Ubilla, M. (2007). Stegomastodon waringi (Mammalia, Proboscidea) from the Late Pleistocene of Northeastern Uruguay. Neues Jahrbuch für Geologie und Paläontologie Abhandlungen, 243 (2): 179-189. http://dx.doi.org/ 10.1127/0077-7749/2007/0243-0179

Alberdi, M.T.; Prado, J.L. \& Salas, R. (2004). The Pleistocene gomphotheres (gomphotheriidae, proboscidea) from Peru. Neues Jahrbuch für Geologie und Paläontologie Abhandlungen, 231: 423-452.

Ameghino, F. (1888). Rápidas diagnosis de algunos mamíferos fósiles nuevos de la República Argentina. Obras Completas, Buenos Aires. Vol. 5, p. 469-480.

Cabrera, A. (1929). Una revisión de los Mastodontes Argentinos. Revista del Museo de La Plata, 32: 61-144.

Campbell, K.E. Jr.; Frailey, C.D. \& Romero-Pittman, L. (2000). The late Miocene gomphothere Amahuacatherium peruvium (Proboscidea: Gomphotheriidae) from Amazonian Peru: implications for the Great American Faunal Interchange. Boletin - Instituto Geológico Minero y Metalúrgico. Serie D, Estudios Regionales, 23: 1-152.

Cione, A.L. \& Tonni, E.P. (2005). Bioestratigrafía basada en mamíferos del Cenozoico superior de la provincia de Buenos Aires, Argentina. In: Geología y Recursos Minerales de la Provincia de Buenos Aires (de Barrio, R.E.; Etcheverry, R.O.; Caballé, M.F. \& Llambias, E., Eds.). XVI Congreso Geológico Argentino. La Plata, 2005. p. 183-200.

Corona, A.; Perea, D.; Toriño, P. \& Goso, C. (2012). Taphonomy, sedimentology and chronology of a fossiliferous outcrop from the continental Pleistocene of Uruguay. Revista Mexicana de Ciencias Geológicas, 29 (2): 514-525.

Ferretti, M. (2008). A review of South American Proboscideans. New Mexico Museum of Natural History \& Science Bulletin, 44: 381-391.

Goso, H. \& Bossi, J. (1966). Cenozoico. In: Geología del Uruguay (Bossi, J., Ed.). Departamento de Publicaciones, Universidad de la República, Montevideo. 469 p.

Gutiérrez, M.; Alberdi, M.T.; Prado, J.L. \& Perea, D. (2005). Late Pleistocene Stegomastodon (Mammalia, Proboscidea) from Uruguay. Neues Jahrbuch fûr Geologie und Paläontologie Monatshefte, 11: 641-662. 
Holland, W.J. (1920). Fossil mammals collected at Pedra Vermelha, Bahia, Brazil, by Gerald A Waring. Annals of the Carnegie Museum, 13: 224-232.

Illiger, C. (1811). Prodromus systematis mammaliumet avium additis terminis zoographisis utriudque clasis. Berlin, C. Salfeld. 301 p.

Linnaeus, C. (1758). Systema naturae per regna tria naturae, secundum classes, ordines, genera, speciescum characteribus, differentiis, synonymes, locis. Editio decima, reformata. Stockholm, Laurentii Salvii. Vol. I, 824 p.

Lucas, S.G. (2013). The palaeobiogeography of South American gomphotheres. Journal of Palaeogeography, 2 (1): 19-40. http://dx.doi.org/10.3724/ SP.J.1261.2013.00015

Lucas, S.G. \& Alvarado, G.E. (2010). Fossil Proboscidea from the Upper Cenozoic of Central America: taxonomy, evolutionary and paleobiogeographic significance. Revista Geológica de América Central, 42: 9-42.

Martínez, S. (1994). Bioestratigrafía (Invertebrados) de la Formación Camacho (Mioceno, Uruguay). Tesis doctoral, Universidad de Buenos Aires, Facultad de Ciencias Exactas y Naturales. 346 p.

Martínez, S. \& Ubilla, M. (2004). El Cuaternario en Uruguay. In: Cuencas sedimentarias de Uruguay, Cenozoico (Veroslavsky, G., Ubilla, M. \& Martínez, S., Eds.). Ediciones DIRAC, Facultad de Ciencias, Montevideo, p. 195-227.

Mones, A. \& Francis, J.C. (1973). Lista de los vertebrados fósiles del Uruguay, II, Mammalia. Comunicaciones Paleontológicas del Museo de Historia Natural de Montevideo, 1 (4): 39-97.

Mothé, D.; Avilla, L.; Cozzuol, M. \& Winck, G. (2012). Taxonomic revision of the quaternary gomphotheres (Mammalia: Proboscidea: Gomphotheriidae) from the South American lowlands. Quaternary International, 276-277: 2-7. http://dx.doi.org/10.1016/j.quaint. 2011.05.018

Mothé, D.; Avilla, L. \& Cozzuol, M. (2013). The South American Gomphotheres (Mammalia, Proboscidea,
Gomphotheriidae): Taxonomy, Phylogeny, and Biogeography. Journal of Mammal Evolution, 20 (1): 23-32.http://dx.doi.org/10.1007/s10914-012-9192-3

Perea, D. \& Martínez, S. (1984). La fauna fósil (Mammalia, Reptilia y Mollusca) de los arroyos Gutiérrez Grande y Chico, Depto. de Río Negro, Uruguay. Boletín de la Sociedad Zoológica del Uruguay (2da. Época), 2: 47-53.

Perea, D. \& Martínez, S. (2004). Estratigrafía del MiocenoPleistoceno en el litoral sur-oeste de Uruguay. In: Cuencas sedimentarias de Uruguay, Cenozoico (Veroslavsky, G., Ubilla, M. \& Martínez, S., Eds.). Ediciones DIRAC, Facultad de Ciencias, Montevideo. p. 105-124.

Pohlig, H. (1912). Sur une vieille mandibule de 'Tetracaulodon ohiotocum' Blum., avec défense in situ. Bulletin de la Société Belge Géologique, 26: 187-193.

Prado, J.L.; Alberdi, M.T.; Azanza, B.; Sánchez, B. \& Frassinetti, D. (2005). The Pleistocene Gomphotheriidae (Proboscidea) from South America. Quaternary International, 126-128: 21-30. http://dx.doi. org/10.1016/j.quaint.2004.04.012

Roselli, L. (1976). Contribución al estudio de la geo paleontología. Departamentos de Colonia y Soriano (República Oriental del Uruguay). Imprenta Cooperativa, Montevideo, $174 \mathrm{p}$.

Ubilla, M. \& Perea, D. (1999). Quaternary Fossil Vertebrates from Uruguay. Quaternary of South America and Antarctic Peninsula, 12: 75-90.

Ubilla, M.; Perea, D.; Rinderknecht, A. \& Corona, A. (2009). Pleistocene mammals from Uruguay: biostratigraphic, biogeographic and environmental connotations. In: Quaternário do Rio Grande do Sul (Ribeiro, A.M., Bauermann, S.G. \& Scherer, Eds.) Sociedade Brasileira de Paleontología, Porto Alegre. p. 217-230.

Ubilla, M.; Rinderknecht, A.; Perea, D. \& Sánchez, A. (2011). Late Pleistocene-Early Holocene mammals of the Santa Lucía Basin (southern Uruguay). Ameghiniana, 48 (4): R131. 